\title{
Alcohol y otras drogas: acciones en busca de soluciones
}

\author{
Antonia Regina Ferreira Furegato
}

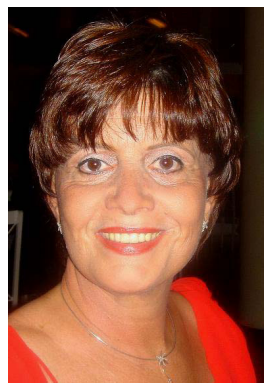

Tanto el uso como el abuso de alcohol y otras drogas afectan significativamente la calidad de vida de los usuarios y de sus familiares, teniendo, además de eso, enorme costo social y económico en todo el mundo. Según la Organización Mundial de la Salud - OMS, la mayoría de los consumidores se encuentra entre 18 y 35 años de edad, estando, por tanto, ese fenómeno asociado al estudio, al trabajo y a la constitución de nuevos grupos familiares ${ }^{(1-2)}$.

Tratando de enfrentar el problema que crece en el uso y las complicaciones, así como en las consecuencias personales y sociales, las autoridades políticas y científicas han aumentado los esfuerzos para explorar: posibles determinantes de ese proceso, componentes de actitudes potencialmente favorables al envolvimiento de la persona con las drogas, comportamientos predictivos de uso, así como la presencia de fuentes de soporte y de apoyo. La Organización de los Estados Americanos - OEA, a través de la Comisión Interamericana para el Control del Abuso de las Drogas - CICAD, viene, desde hace más de seis anos, trabajando para crear alianzas con instituciones de enseñanza e investigación en el área de la salud, especialmente en la enfermería, con el objetivo de preparar a los profesionales de la salud para mejorar la calidad del cuidado en todos los niveles de atención a los usuarios ${ }^{(2-3)}$.

En esta edición especial de la Revista Latino-Americana de Enfermería, se reúnen los textos producidos por profesionales de la salud de América Latina y de África, que participaron del Programa CICAD, coordinado por la Escuela de Enfermería de Ribeirao Preto de la Universidad de Sao Paulo, y realizaron investigaciones que aportan datos e informaciones epidemiológicas, así como experiencias de estudiantes de enfermería, medicina y otros, en contacto con las drogas. Algunos artículos traen informaciones de las vivencias y percepciones sobre ese fenómeno, destacándose factores protectores y de riesgo, y otros, informaciones sobre estrategias de enfrentamiento que están siendo implementadas en diferentes países, tales como terapia comunitaria, intervenciones breves, programas de rehabilitación específicos y estrategias de prevención y vigilancia.

Se observa que la población de los estudios presentados, publicados en este fascículo, fue constituida por adolescentes y adultos jóvenes, con la intención de conocer el problema en esos grupos poblacionales e identificar aproximaciones terapéuticas.

En esa línea de investigación, se destaca el estudio realizado con 5.581 estudiantes, de 27 escuelas, en Alemania, en donde se analizó el efecto del contacto de los estudiantes con una serie de 50 películas, que contenían escenas de uso de alcohol, y cuyos resultados evidenciaron que existe una relación entre el tiempo de exposición a las escenas de uso de alcohol y el consumo (binge drinking $\geq 5$ dosis), sin el conocimiento de los padres. El estudio fue controlado por edad, género, desempeño y nivel de escolaridad, uso en la familia, beber con los amigos, los cuales, normalmente, son considerados factores predictores del uso de alcohol. El estudio concluye que los medios 
de comunicación contribuyen para el uso del alcohol entre los jóvenes, ya que los mensajes ejercen influencia en sus creencias y en sus comportamientos ${ }^{(4)}$.

Las familias, directamente afectadas por el cambio de comportamiento del joven que pasa a usar alcohol u otras drogas, no han encontrado, en los servicios de salud y tampoco en otras instancias de la sociedad, la orientación y el soporte necesarios para enfrentar las dificultades y los efectos negativos, asociados a esa situación.

La creación de los Núcleos de Atención Psicosocial - CAPSad II, y las Unidades de Internación especializadas, por determinación del Ministerio de la Salud, han buscado minimizar esa carencia (Ordenanza GM nº 336 de $19 / 2 / 2002)^{(5)}$.

Los profesionales del área de la salud, entretanto, formados en el modelo biológico de atención, encuentran dificultades para atender al usuario de alcohol y otras drogas en la perspectiva psicosocial, ya que esa atención envuelve diferentes dimensiones que ultrapasan la medicalización, no se restringiendo a acciones directas de causa y efecto $^{(6)}$.

La IV Conferencia Nacional de Salud Mental, realizada en Brasilia, DF, Brasil, en 2010, que prestó atención a los avances y desafíos de ese escenario, destacó el compromiso de profundizar y madurar la articulación política entre el campo de la salud mental y las instituciones de control social, en los 3 niveles de la gestión del Sistema Único de Salud - SUS. En sus recomendaciones, asumió el compromiso de hacer efectiva la política de reducción de daños en la rede de atención psicosocial, fortaleciendo prácticas territoriales y la construcción de redes de apoyo, en contraposición al modelo enfocado en la abstinencia, en la moralización, penalización y criminalización del usuario de alcohol y otras drogas. Eso implica en un compromiso de sustentabilidad de la propuesta, instituyendo mecanismos para fortalecer la integración de la atención básica con los CAPS, además de crear camas en hospitales generales y fortalecer las acciones de promoción de la salud, prevención y reducción de daños. Se comprometió, también, a promover acciones junto a la sociedad, objetivando ofrecer conocimientos, disminuir los prejuicios y el estigma asociado al consumo de alcohol y otras $\operatorname{drogas}^{(7)}$.

\section{Referencias}

1. World Health Organization (USA). The world Health Report. Working Together for Health. Geneva: WHO; 2006.

2. Ventura CAA, Brands B, Adiaf E, Giesbrecht N, Simich L, Wright MGM, Ferreira OS. Políticas e leis sobre drogas ilícitas no Brasil e a perspectiva de familiares e pessoas próximas a usuários de drogas: estudo na cidade de Riberião Preto, São Paulo, Brasil. Rev. Latino-Am. Enfermagem. 2009;17(especial):810-6.

3. CICAD-CAMH ( 2006-2009). Informes finales del Programa internacional en investigación para professionales de la salud y areas relacionadas para estudiar el fenomeno de las drogas en America Latina y Caribe. Washington (DC), Toronto (CAN); 2009.

4. Hanewinkel R,Tasky SE, Sargent JD. Exposure to alcohol use in motion pictures and teen drinking in Germany. Int J Epidemiol. 2007;36(5):1068-77.

5. Ministério da Saúde (BR). Secretaria Executiva. Secretaria de Atenção à Saúde. Legislação em saúde mental 1990 a 2004. Brasília: Ministério da Saúde; 2004.

6. Cruz MS, Silva Filho JF. A formação de profissionais para a assistência de usuários de drogas e a constituição de um novo habitus de cuidado. J Bras Psiquiatr. 2005;54(2):120-6.

7. Ministério da Saúde (BR). Relatório Final da IV Conferência Nacional de Saúde Mental. Brasília: Ministério da Saúde; 2010.

Antonia Regina Ferreira Furegato es Miembro de la Comisión Editorial de la Revista Latino-Americana de Enfermería, y Profesora Titular de la Escuela de Enfermería de Ribeirao Preto de la Universidad de Sao Paulo, Centro Colaborador de la OMS para el Desarrollo de la Investigación en Enfermería, SP, Brasil. E-mail: furegato@eerp.usp.br. 